\title{
Quantization of Poisson pencils and generalized Lie algebras
}

\author{
D.Gurevich \\ Université de Valenciennes, \\ 59304 Valenciennes, France, \\ V.Rubtsov \\ Centre de Mathématiques, Ecole Polytechnique, \\ 91128 Palaiseau, France \\ and ITEP, Bol.Tcheremushkinskaya 25, \\ 117259 Moscow, Russia
}

\begin{abstract}
We describe two types of Poisson pencils generated by a linear bracket and a quadratic one arising from a classical R-matrix. A quantization scheme is discussed for each. The quantum algebras are represented as the enveloping algebras of "generalized Lie algebras".
\end{abstract}

\section{Introduction}

It is well-known that Poisson pencils play an important role in the theory of integrable dynamical systems. Recall that a Poisson pencil is a linear space of Poisson brackets generated by two of them

$$
\{,\}_{a, b}=a\{,\}_{1}+b\{,\}_{2} .
$$

In this case the brackets $\{,\}_{1}$ and $\{,\}_{2}$ are called compatible.

However it was not clear what was a proper quantum analog of Poisson pencil (1) in general. In the present paper we propose certain candidates for 
this role in a particular case when one of the brackets (say $\{,\}_{1}$ ) is linear and the other $\left(\{,\}_{2}\right)$ is quadratic and defined by a classical "canonical" R-matrix (2).

Actually we consider two types of Poisson pencils (1) connected with R-matrices and discuss a way to quantize them.

Let us describe them but first we want to make a few remarks about our notations.

All manifolds (or varieties) will be considered over the field $k=\mathbf{R}$. By Fun $(M)$ we always mean the space of polynomials in the case when $M=V$ is a linear space or the space of their restrictions to $M$ if $M$ is embedded as an algebraic variety in $V$. In the last case, we say that a Poisson bracket is linear (quadratic) if the Poisson bracket of the restrictions of two linear functions is the restriction of a linear (quadratic) function.

Note that all classical and quantum objects under consideration have natural holomorphic analogs over the field $k=\mathbf{C}$.

Type 1. Let $\mathfrak{g}$ be a Lie algebra and $\rho: \mathfrak{g} \rightarrow V e c t(M)$ be a representation of $\mathfrak{g}$ in the space of vector fields on a manifold $M$. Let us assign to an element $R \in \wedge^{2}(\mathfrak{g})$ a bi-vector field $\rho^{\otimes 2}(R)$ and introduce a bracket

$$
\{f, g\}_{R}=\mu<\rho^{\otimes 2}(R), d f \otimes d g>.
$$

Hereafter $\mu$ denotes the usual commutative multiplication in $F u n(M)$. We are interested in the cases when this bracket is Poisson.

If it is, the manifold $M$ is equipped with another Poisson bracket $\{$, and the elements of $\operatorname{Im}(\rho)$ preserve the latter bracket, i.e.,

$$
\rho(X)\{,\}=\{,\}(\rho(X) \otimes i d+i d \otimes \rho(X)), \forall X \in \mathfrak{g}
$$

then we have a Poisson pencil (1) with $\{,\}_{1}=\{$,$\} and \{,\}_{2}=\{,\}_{R}$.

The case under detailed examination is the following: $\mathfrak{g}$ is a simple Lie algebra, $M=\mathcal{O}_{x}$ is the orbit in $\mathfrak{g}^{*}$ of a highest weight element $x \in \mathfrak{g}^{*}$ (h.w. orbit for brevity) equipped with the Kirillov-Kostant-Souriau bracket $\{\}=,\{,\}_{K K S}, R$ is assumed to be R-matrix (2) and $\rho=a d^{*}$.

Type 2. Let $\mathfrak{g}$ be a classical simple Lie algebra (i.e., one of the following algebras $s l(n), s o(n), s p(n))$. Consider the Sklyanin-Drinfeld bracket $\{,\}_{S D}$ (refered also as Poisson-Lie) defined on the corresponding group $G$ as follows

$\{f, g\}_{S D}=\{f, g\}_{l}-\{f, g\}_{r},\{f, g\}_{r, l}=\mu<\rho_{r, l}^{\otimes 2}(R), d f \otimes d g>, f, g \in F u n(G)$, 
where $\rho_{r}\left(\rho_{l}\right)$ is the representation of the Lie algebra $\mathfrak{g}$ in the space $\operatorname{Fun}(G)$ by the left- (right-) invariant vector fields and $R$ is an R-matrix (2).

Let us consider an embedding of the group $G$ in the matrix algebra $\operatorname{Mat}(n)$. Then the bracket $\{,\}_{S D}$ can be extented to the bracket defined on the space $\operatorname{Fun}(\operatorname{Mat}(n))$ in a natural way but it is Poisson iff $\mathfrak{g}=\operatorname{sl}(n)$. In this case the extended bracket will be denoted $\{,\}_{2}$.

Note that the bracket $\{,\}_{2}$ is quadratic with respect to the basis given by the matrix elements. Linearization of this bracket gives rise to a Poisson bracket (denoted $\{,\}_{1}$ ) compatible with the bracket $\{,\}_{2}$. Thus we have a Poisson pencil defined in the space Fun $(\operatorname{Mat}(n))$ and generated by the brackets $\{,\}_{1,2}$.

Finally we have two types of Poisson pencils arising from R-matrices. The main difference between them is following. For the first type Poisson pencil we start with a linear bracket and construct the quadratic one by means of a classical R-matrix. In contrary, the linear bracket of the second type Poisson pencil is appeared as a linearization of a given quadratic bracket. Unfortunately we do not know any scheme which could include both types.

Note that integrability of the Toda chain is connected with the last type Poisson pencils.

Let us say a few words about the quantization of Poisson pencils of both types. Note that a quantization scheme of the first type Poisson pencils in the case when $R$ is a classical (non-modified, cf. below) R-matrix was proposed in GRZ. The case when $R$ is R-matrix (2) and $M$ are orbits in $\mathfrak{g}^{*}$ of a special type was examined in [DG1], [DS2].

The algebras quantizing the Poisson pencils under consideration (quantum algebras for short) can be described as follows. We describe the quantum algebras corresponding to the brackets $\{,\}_{2}$ in terms of graded quadratic algebras:

For the second type Poisson pencils this algebra is q-deformed symmetric algebra of the space $\operatorname{Mat}(n)^{*}$. For the first type ones it is a q-deformed g.q.a. which corresponds due to Kostant's theorem (cf. [LT]) to the h.w. orbit in $\mathfrak{g}^{*}$.

To quantize the whole Poisson pencil (2) we describe the correspoding

\footnotetext{
${ }^{1}$ Let us precise that we call a filtered quadratic algebra (f.q.a.) an algebra of the form $T(V) /\{I\}$ where $V$ is a linear space and $I \subset V^{\otimes 2} \oplus V \oplus k$. Hereafter we denote $T(V)$ the free tensor algebra of a linear space $V$ and $\{I\}$ denotes the ideal generated by a set $I$. If $I \subset V^{\otimes 2}$ we call the corresponding algebra graded quadratic (g.q.a.).
} 
quantum algebras in terms of f.q.a. which look like the enveloping algebras of the Lie algebras (with a parameter $h$ introduced in the Lie bracket). Thus we have two parameter quantum algebras $A_{h, q}$ (a parameter $q=e^{\nu}$ is one of "braiding" and another parameter $h$ appears while deforming a commutative algebra of functions to an enveloping algebra). The algebras $A_{h, q}$ will be called of the first or second type accordingly to the type of the initial Poisson pencil. (Let us emphasize that unlike our quantum algebras the famous quantum groups $U_{q}(\mathfrak{g})$ are not f.q.a.)

In this connection a natural question arises : whether it is possible to represent the latter algebra itself as the enveloping algebra of a q-deformed or "generalized" (in some sense) Lie algebra structure (in particular, what is a proper analog of Jacobi identity in the general case) ?

There were number of attempts to find a proper q-analog of the Lie algebras. In particular, objects of such a type have been considered in [DOS] (cf. also the references therein). In [W] some generalized Lie structures were introduced in terms of "quantum differential calculus". A version of "quantum" and "braided" Lie algebras were proposed by Sh.Majid [M]. However, in all these papers the following question was ignored: whether the corresponding enveloping algebra is a flat deformation [2] of the initial classical counterpart.

We propose our version of "deformed Lie algebras", called in the paper "generalized Lie algebras", using as a criterium the flatness of deformation of the enveloping algebras. (In the present paper we consider only algebras lying in quasiclassical categories, i.e. whose braiding operator is a deformation of the flip though the below definition is valuable in the general case). In this case we have two parameter family of associative algebras (the sense of the parameters is explained above) and as a quasiclassical limit of this family we have a Poisson pencil. Thus the existence of a Poisson pencil is a necessary condition for the "reasonable" from the above point of view deformation of a ordinary Lie algebra structure. (Let us emphasize that this is not a

\footnotetext{
${ }^{2}$ One says that a deformation from $A_{0}$ to $A_{h}$ is flat if $A_{h} / h A_{h}$ coincides with $A_{0}$ and $A_{h}$ is a free $k[[h]]$-module. As for a deformation of g.q.a. $A_{0} \rightarrow A_{h}$ it is flat iff $\operatorname{dim}_{k[[h]]} A_{h}^{p}=\operatorname{dim} A_{0}^{p}$ where $A_{h}^{p}$ is an homogeneous component of $A_{h}$ of degree $p$. A deformation of a g.q.a. $A_{0}$ to f.q.a. $A_{h}$ is flat if there exists a natural isomorphism of the PBW type between $\operatorname{Gr} A_{h}$ and $A_{0}$.

${ }^{3}$ Note that there exist non-quasiclassical categories, e.g, super-categories. Other examples of non-quasiclassical rigid tensor or quasitensor categories can be constructed by means of non-quasiclassical solutions of the QYBE, introduced in [G2], G3].
} 
deformation of one Lie algebra to another and the usual deformation theory can not be applied here.)

Thus, observing that the SD bracket (extended to Fun(Mat(2))) is not compatible with the linear bracket correspondind to the Lie algebra $g l(2)$ it is easy to show that there does not exist any "reasonable" deformed version of $g l(2)$ in the frames of the second type construction. (As for the first type deformation of the Lie algebra $s l(2)$, cf. [DG1).

In any case if we want to get such a deformation of a Lie algebra $\mathfrak{g}$ that the deformation of the corresponding enveloping algebras is flat we should restrict ourselves to the h.w. orbit in $\mathfrak{g}^{*}$ instead of the initial symmetric algebra of the space $V=\mathfrak{g}$ (for the type 1 ) or take as the initial Lie algebra one given by the formula (5) (for the type 2). In the latter case we can obtain a deformation of the Lie algebra which forms a double Lie algebra with $g l(n)$.

The paper is organized as follows. In next Section we introduce two type of Poisson pencils connected to R-matrix (2). In Section 3 we describe a scheme of their quantization. The role of "generalized Lie algebras" in the quantization procedure is discussed in Section 4. An example of such a generalized Lie algebra arising from a second type Poisson pencil is given in the last Section.

\section{Two types of Poisson pencils}

Recall that an element $R \in \wedge^{2}(\mathfrak{g})$ where $\mathfrak{g}$ is a Lie algebra is called a classical $R$-matrix if the element

$$
[[R, R]]=\left[R^{12}, R^{13}\right]+\left[R^{12}, R^{23}\right]+\left[R^{13}, R^{23}\right],
$$

which always lies in $\wedge^{3}(\mathfrak{g})$, is equal to 0 . We call $R$ a modified $\mathrm{R}$-matrix if it satisfies the classical modified YBE, i.e., the element $[[R, R]]$ is $\mathfrak{g}$-invariant.

Type 1. Given a Poisson bracket $\{$,$\} on a manifold M$, consider the space $\operatorname{Vect}(M,\{\}$,$) of the vector fields X \in V \operatorname{ect}(M)$ preserving this bracket. It is evident that they form a Lie algebra with respect to the usual Lie bracket

$$
X \otimes Y \in \operatorname{Vect}(M,\{,\})^{\otimes 2} \rightarrow[X, Y]=X Y-Y X .
$$

Let $\rho: \mathfrak{g} \rightarrow \operatorname{Vect}(M,\{\}$,$) be a morphism of a finite dimensional Lie$ algebra $\mathfrak{g}$ in the space $\operatorname{Vect}(M,\{\}$,$) equipped with the above Lie structure.$ 
Let us fix an element $R \in \wedge^{2}(\mathfrak{g})$. Consider the bracket $\{,\}_{R}$ corresponding to this element. If it is Poisson we have a Poisson pencil (1) since the brackets $\{$,$\} and \{,\}_{R}$ are always compatible.

It is, e.g., if $R$ is a classical non-modified R-matrix. Let us assume now that $\mathfrak{g}$ is a simple Lie algebra and $R$ is a "canonical" modified R-matrix

$$
R=\sum_{\alpha \in \Delta_{+}} X_{\alpha} \wedge X_{-\alpha} \in \wedge^{2} \mathfrak{g} .
$$

Here $\left\{H_{\alpha}, X_{\alpha}, X_{-\alpha}\right\}$ is the Cartan-Weyl system in the Chevalley normalization and $\Delta_{+}$is the set of the positive roots of $\mathfrak{g}$ (other normalizations of this R-matrix are possible as well).

Then the bracket $\{,\}_{R}$ is Poisson only on certain manifolds (we say that such a manifold $M$ is of R-matrix type). All symmetric homogeneous $G$-spaces $M=G / H$ and the h.w. orbits in any linear space possessing a $G$-module structure are of R-matrix type (cf. [DGM], GP]).

Restricting ourselves to orbits $M=\mathcal{O}_{x}$ in $\mathfrak{g}^{*}$ of R-matrix type we get a family of examples of Poisson pencils taking as the bracket $\{,\}_{1}$ the Kirillov-Kostant-Souriau bracket $\{,\}_{K K S}$.

Other examples can be constructed as follows. Fix an even-dimensional linear space $V$ equipped with a skew-symmetric non-degenerated pairing $<$ ,$>: V^{\otimes 2} \rightarrow k$ and the Lie algebra $\mathfrak{g}=s p(V)$ of linear transformations preserving this form $(<X u, v\rangle+\langle u, X v\rangle=0)$. Introduce in the space Fun $\left(V^{*}\right)$ a Poisson bracket $\{,\}_{1}$ in the following way : for linear functions $f, g \in V$ we set $\{f, g\}_{1}=<f, g>$. Then taking as $R$ the R-matrix (2) corresponding to the Lie algebra $s p(V)$, (which is isomorphic to the algebra $s p(n)$ ), we get a quadratic Poisson bracket $\{,\}_{R}$ (it is Poisson since $V \backslash 0$ is the h.w. orbit) and this bracket is compatible with the bracket $\{,\}_{1}$.

The following proposition is obvious.

Proposition 1 If $\rho(\mathfrak{g})$ falls into the space of (locally) Hamiltonian vector fields then any symplectic leave of the bracket $\{,\}_{R}$ lies in some leave of the bracket $\{,\}_{1}$.

Type 2. Let us assume now that $G$ is a classical simple Lie group, i.e., one of the following Lie groups $S L(n), S O(n), S p(n)$ and $R$ is R-matrix (2). Let us consider on $G$ the SD bracket.

Note that this bracket can be represented in the following form

$$
\left\{L_{1} \otimes, L_{2}\right\}_{S D}=\left[R_{\rho}, L_{1} \circ L_{2}\right], L_{1}=L \otimes i d, L_{2}=i d \otimes L .
$$


Here $L=\left(a_{i}^{j}\right), 1 \leq i, j \leq n=\operatorname{dim} V$ is a matrix with the matrix elements $a_{i}^{j}$ (the low index labels rows), $\rho: \mathfrak{g} \rightarrow \operatorname{End}(V)$ is the fundamental vector representation and $R_{\rho}=\rho^{\otimes 2}(R)$. This bracket was originated by E. Sklyanin namely in the form (3).

One can extend this bracket to the space Fun $(\operatorname{Mat}(n))$ using the formula (3). This means that the bracket $\{f, g\}_{S D}$ is well-defined for any functions $f, g \in \operatorname{Fun}(\operatorname{Mat}(n))$. However the extended bracket is Poisson iff $\mathfrak{g}=\operatorname{sl}(n)$. We denote the extended bracket $\{,\}_{2}$.

In what follows we consider in the frames of the second type construction only the case $\mathfrak{g}=\operatorname{sl}(n)$.

Let us linearize now the bracket $\{,\}_{2}$ (the resulting bracket will be denoted $\left.\{,\}_{1}\right)$. The linearization can be defined as follows. In the r.h.s. of the formula (3) we replace the matrix $L$ by $L+\lambda i d$ (i.e., we substitute $a_{i}^{j}+\lambda \delta_{i}^{j}$ instead of $a_{i}^{j}$ ) and take only linear term in $\lambda$. In a compact form the result can be represented as follows

$$
\left\{L_{1}, L_{2}\right\}_{1}=\left[R_{\rho}, L_{1}+L_{2}\right]
$$

Then the latter bracket is Poisson. Moreover, the brackets $\{,\}_{1}$ and $\{,\}_{2}$ are compatible.

This fact can be deduced from [S] but it is easy to prove it directly observing that the r.h.s. of (4) after the above substitution does not contains any terms quadratic in $\lambda$.

Note that a linearizarion of the SD bracket on any group $G$ gives rise according to the Drinfeld construction [D] to a Lie structure in $\mathfrak{g}^{*}$ (which forms a so-called Manin triple toghether with the initial Lie algebra $\mathfrak{g}$ ). So the corresponding (linear) Poisson-Lie bracket is well-defined in the space $\operatorname{Fun}(\mathfrak{g})$.

As for the case when $\mathfrak{g}=\operatorname{sl}(n)$ the bracket (4) is an extention of the latter bracket to the space Fun $(\operatorname{Mat}(n))$. In this case we have two Poisson brackets $\{,\}_{1,2}$ defined simultaneously in the space Fun $(\operatorname{Mat}(n))$, (the first of them can be reduced to Fun $(\operatorname{sl}(n))$ and the second one to $\operatorname{Fun}(S L(n))$.

Remark 1 There exist (at least) two schemes to construct integrable $d y$ namical systems. The first (Magri-Lenart scheme) deals with bi-Hamiltonian systems. (Recall that a dynamical system is called bi-Hamiltonian if it is Hamiltonian with respect to two different Poisson structures.) 
Another scheme was developped by Adler, Kostant and Symes. In $R$ matrix interpretation, proposed by the St-Petersbourg school, it deals with two Lie structures defined in the same linear space (so-called double Lie algebras). We refer the reader to $S$ for details.

Note that for the second type Poisson pencils the symplectic leaves of the first bracket do not lie in the leaves of the second one. This condition is necessary for constructing the integrable dynamic systems.

Let us give now an explicit form of the second type Poisson brackets $\{,\}_{1,2}$ under consideration.

The multiplication table for the second Poisson bracket is

$$
\begin{gathered}
\left\{a_{k}^{i}, a_{k}^{j}\right\}_{2}=a_{k}^{i} a_{k}^{j},\left\{a_{i}^{k}, a_{j}^{k}\right\}_{2}=a_{i}^{k} a_{j}^{k}, i<j ; \\
\left\{a_{i}^{l}, a_{k}^{j}\right\}_{2}=0,\left\{a_{i}^{j}, a_{k}^{l}\right\}_{2}=2 a_{i}^{l} a_{k}^{j}, i<k, j<l .
\end{gathered}
$$

It is not difficult to deduce from this the multiplication table for the first Poisson bracket:

$$
\begin{gathered}
\left\{a_{k}^{i}, a_{k}^{j}\right\}_{1}=\delta_{k}^{i} a_{k}^{j}+a_{k}^{i} \delta_{k}^{j},\left\{a_{i}^{k}, a_{j}^{k}\right\}_{1}=\delta_{i}^{k} a_{j}^{k}+a_{i}^{k} \delta_{j}^{k}, i<j ; \\
\left\{a_{i}^{l}, a_{k}^{j}\right\}_{1}=0,\left\{a_{i}^{j}, a_{k}^{l}\right\}_{1}=2\left(\delta_{i}^{l} a_{k}^{j}+a_{i}^{l} \delta_{k}^{j}\right), i<k, j<l .
\end{gathered}
$$

It is useful to represent the latter bracket under the following form

$$
\left\{a_{i}^{j}, a_{k}^{l}\right\}_{1}=\left\{\mathbf{R}\left(a_{i}^{j}\right), a_{k}^{l}\right\}_{g l}+\left\{a_{i}^{j}, \mathbf{R}\left(a_{k}^{l}\right)\right\}_{g l} .
$$

Here $\{,\}_{g l}$ is the Poisson-Lie bracket corresponding to the Lie algebra $g l(n)$ (namely, $\left\{a_{i}^{j}, a_{k}^{l}\right\}_{g l}=a_{i}^{l} \delta_{k}^{j}-a_{k}^{j} \delta_{i}^{l}$ ) and $\mathbf{R}: W \rightarrow W$ is an operator defined in the space $W=\operatorname{Span}\left(a_{i}^{j}\right)$ as follows $\mathbf{R}\left(a_{i}^{j}\right)=\operatorname{sign}(j-i) a_{i}^{j}$ (we assume that $\operatorname{sign}(0)=0)$.

Actually we have equipped the space $W$ with two Lie structures. The first one is $g l(n)$ and the seconde corresponds to the Poisson bracket $\{,\}_{1}$. Thus we have a double Lie algebra in the space $W$ (our construction coincides with one from [S] up to a factor). 


\section{Quantization scheme}

In the present section we discuss the quantization scheme for both types of the Poisson pencils (2). This scheme consists of two steps. On the first step we describe the quantum algebra for the bracket $\{,\}_{2}$. This algebra can be represented as a g.q.a for both types. However, there exists a difference between them. If for the second type Poisson pencil we deform the symmetric algebra of the initial space, for the first type we deal with a deformation of the g.q.a. coressponding to h.w. orbits in $\mathfrak{g}^{*}$. Meanwhile for the both cases the deformations are flat.

On the second step we are looking for linear terms converting the mentioned g.q.a. into a f.q.a. in such a way that two properties are fulfiled:

1. the deformation from the g.q.a. constructed before to f.q.a. is flat;

2. the corresponding quasiclassical limit of this deformation coincides with the given Poisson pencil.

Type 1. Let us fix a simple Lie algebra $\mathfrak{g}$ and the corresponding quantum group $U_{q}(\mathfrak{g})$. Let us consider a linear space $V$ equipped with a $\mathfrak{g}$-module structure $\rho: \mathfrak{g} \rightarrow \operatorname{End}(V)$ and endow it with a structure of $U_{q}(\mathfrak{g})$-module $\rho_{q}: U_{q}(\mathfrak{g}) \rightarrow \operatorname{End}(V)$. We assume that $\rho_{q} \rightarrow \rho$ when $q$ goes to 1 .

Let us represent $V^{\otimes 2}=\oplus V_{\beta}$ as a direct sum of the isotypical $\mathfrak{g}$-modules ( $\beta$ is the h.w. of the module $V_{\beta}$ ). If $\alpha$ is the h.w. of $V$ as $\mathfrak{g}$-module then in the above decomposition there is an irreducible $\mathfrak{g}$-module $V_{2 \alpha}$. Let us set $\bar{I}_{-}=\oplus_{\beta \neq 2 \alpha} V_{\beta}$ and $\bar{I}_{+}=V_{2 \alpha}$. Reproducing this procedure in the category of $U_{q}(\mathfrak{g})$-modules we can introduce q-counterparts $\bar{I}_{ \pm}^{q}$ of the spaces $\bar{I}_{ \pm}$(cf. [DG2 for details).

Note that in virtue of the Kostant theorem (cf. [[T]]) the function algebra on the h.w. orbit in $V^{*}$ is just the algebra $A_{0,1}=T(V) /\left\{\bar{I}_{-}\right\}$.

As was shown in [DS1] the deformation from the algebra $A_{0,1}$ to its qdeformed counterpart $A_{0, q}=T(V) /\left\{\bar{I}_{-}^{q}\right\}$ is flat.

Remark 2 It is possible to define a q-analog of the symmetric algebra of the space $V$ in a similar way but it is not, in general, a flat deformation of the initial object.

It is not difficult to see that the quasiclassical term of the deformation from $A_{0,1}$ to $A_{0, q}$ coincides up to a factor depending on a normalization of the parameter with the R-matrix bracket defined on the h.w. orbit in $V^{*}$ 
(cf. also [DG2]). Thus the algebra $A_{0, q}$ is the quantum counterpart for the Poisson bracket $\{,\}_{R}$ defined on the h.w. orbits in $V^{*}$.

In a particular case when $V=\mathfrak{g}(\mathfrak{g}$-module structure of $\mathfrak{g}$ is defined by ad-action) we are looking for a futher (non-homogeneous) deformation of the algebra $A_{0, q}$. The latter deformation will be realized by means of the so-called $q$-Lie bracket. In what follows we set $V=\mathfrak{g}$.

Let $\alpha$ be the h.w. of $\mathfrak{g}$ as $\mathfrak{g}$-module. Then in the decomposition $V^{\otimes 2}=$ $\oplus V_{\beta}$ where $V=\mathfrak{g}$ there exists an unique irredicible $\mathfrak{g}$-module $V_{\alpha}$ belonging to the space $I_{-}$.

Let $V_{\alpha}^{q}$ be its analog in the category of $U_{q}(\mathfrak{g})$-modules (i.e. the term in the decomposition of $V^{\otimes 2}$ in the category of $U_{q}(\mathfrak{g})$-modules which is a deformation of the $\mathfrak{g}$-module $V_{\alpha}$, cf. [DG2 for details). Then we introduce $q$-Lie bracket $[,]_{q}: V^{\otimes 2} \rightarrow V$ by setting

$$
\left.[,]_{q}\right|_{V_{\beta}^{q}}=0 \text { if } V_{\beta}^{q} \neq V_{\alpha}^{q} \text { and }[,]_{q}: V_{\alpha}^{q} \rightarrow V
$$

is a morphism in the category of $U_{q}(\mathfrak{g})$-modules (thus the bracket is defined up to a factor).

Let us consider the algebra

$$
A_{h, q}=T(V) /\left\{\oplus_{\beta \neq 2 \alpha} \operatorname{Im}\left(i d-h[,]_{q}\right) V_{\beta}^{q}\right\} .
$$

It is very plausible that the deformation from $A_{0, q}$ to $A_{h, q}$ is flat. Then the deformation from $A_{0,1}$ to $A_{h, q}$ is flat as well.

Setting in the two parameter family $h=a \hbar, q=e^{b \hbar}$ and looking for the Poisson bracket corresponding to this deformation one can reveal a bracket from the pencil (1). Thus, the family of algebras $A_{h, q}$ is the quantum object for the pencil (1) of the first type defined on the h.w. orbit in $\mathfrak{g}^{*}$.

Type 2. Let us consider the base $\left\{a_{i}^{j}\right\}$ in the algebra Fun $(\operatorname{Mat}(n))$ consisting of the matrix elements. We identify the spaces Fun $(\operatorname{Mat}(n))$ and $A_{0,1}=T(W) /\left\{I_{-}\right\}$where $I_{ \pm}$is the subspace of symmetric (skew) elements in $W^{\otimes 2}$. Recall that $W=\operatorname{Span}\left(a_{i}^{j}\right)$.

A quantum (or q-) analog of this space can be described as follows. Let us consider a solution $S=S_{V}: V^{\otimes 2} \rightarrow V^{\otimes 2}$ of the quantum Yang-Baxter equation (QYBE)

$$
S^{12} S^{23} S^{12}=S^{23} S^{12} S^{23}, S^{12}=S \otimes i d, S^{23}=i d \otimes S
$$


where $S=\sigma \rho^{\otimes 2}(\mathcal{R}), \mathcal{R}$ is a quantum universal R-matrix corresponding to the quantum group $U_{q}(\mathfrak{g}), \sigma$ is the flip and $\rho: U_{q}(\mathfrak{g}) \rightarrow \operatorname{End}(V)$ is the vector fundamental representation.

Let us note that in the case under consideration $(\mathfrak{g}=s l(n))$ the operator $S$ is of the "Hecke type", i.e., it possesses two eigenvalues. More precisely, it has the following form

$$
S\left(a_{i} \otimes a_{j}\right)=(q-1) \delta_{i, j} a_{i} \otimes a_{j}+a_{j} \otimes a_{i}+\sum_{i<j}\left(q-q^{-1}\right) a_{i} \otimes a_{j},
$$

where $\left\{a_{i}\right\}$ is a base in the space $V$.

There exists a natural way to introduce an operator $S_{W}: W^{\otimes 2} \rightarrow W^{\otimes 2}$ which satisfies the QYBE setting $S_{W}=S \otimes S^{*-1}$ where $S^{*}: V^{* \otimes 2} \rightarrow V^{* \otimes 2}$ is defined with respect to the pairing

$$
<x \otimes y, a \otimes b>=<x, a><y, b>, x, y \in V, a, b \in V^{*} .
$$

Actually we treate the space $W$ as $V \otimes V^{*}$ and assume that the spaces $V$ and $V^{*}$ commut with each other in the ordinary senset.

In the base $\left\{a_{i}^{j}\right\}$ the operator $S_{W}$ is of the form

$$
S_{W}\left(a_{i}^{k} \otimes a_{j}^{l}\right)=S_{i j}^{m n} S_{p q}^{-1 k l}\left(a_{m}^{p} \otimes a_{n}^{q}\right) \text { where } S\left(a_{i} \otimes a_{j}\right)=S_{i j}^{k l} a_{k} \otimes a_{l} .
$$

Let us point out an important property of the operator $S_{W}$ : it possesses 1 as an eigenvalue. This is the reason why it is natural to introduce a deformed analog of the (skew)symmetric subspace of the space $W^{\otimes 2}$, setting

$$
I_{-}^{q}=\operatorname{Im}\left(S_{W}-i d\right), I_{+}^{q}=\operatorname{Ker}\left(S_{W}-i d\right), A_{0, q}=T(W) /\left\{I_{-}^{q}\right\} .
$$

The explicit form of these spaces is the following:

$$
\begin{gathered}
I_{-}^{q}=\operatorname{Span}\left(a_{k}^{i} a_{k}^{j}-q a_{k}^{j} a_{k}^{i}, a_{i}^{k} a_{j}^{k}-q a_{j}^{k} a_{i}^{k}, i<j ;\right. \\
\left.a_{i}^{l} a_{k}^{j}-a_{k}^{j} a_{i}^{l}, a_{i}^{j} a_{k}^{l}-a_{k}^{l} a_{i}^{j}-\left(q-q^{-1}\right) a_{k}^{j} a_{i}^{l}, i<k, j<l\right)
\end{gathered}
$$

and

$$
\begin{gathered}
I_{+}^{q}=\operatorname{Span}\left(\left(a_{i}^{k}\right)^{2}, q a_{k}^{i} a_{k}^{j}+a_{k}^{j} a_{k}^{i}, q a_{i}^{k} a_{j}^{k}+a_{j}^{k} a_{i}^{k}, i<j ;\right. \\
\left.a_{i}^{j} a_{k}^{l}+a_{k}^{l} a_{i}^{j}, a_{i}^{l} a_{k}^{j}+a_{k}^{j} a_{i}^{l}+\left(q-q^{-1}\right) a_{i}^{j} a_{k}^{l}, i<k, j<l\right) .
\end{gathered}
$$

\footnotetext{
${ }^{4}$ Let us remark that there exists another way to extend the operator $S$ to the space $V \otimes V^{*}$ regarding $V^{*}$ as dual to $V$ in the rigid category generated by $V$ (cf. [G3]).
} 
Proposition 2 ([DS1]) The deformation from $A_{0,1}$ to $A_{0, q}$ is flat.

Let us emphasize that considering the operators $S$ which correspond to other classical simple algebras (and possess in this case 3 eigenvalues) one can introduce in a similar way the spaces $I_{ \pm}^{q}$. However the deformation from $A_{0,1}$ to $A_{0, q}=T(W) /\left\{I_{-}^{q}\right\}$ is not flat in this case.

It is easy to see that the Poisson bracket corresponding to the above deformation coincides up to a factor with $\{,\}_{2}$. Thus we can consider the algebra $A_{0, q}$ as a resulting object for the first step of the quantization for the second type Poisson pencil.

As for a two-parameter family of associative algebras $A_{h, q}$ quantizing the whole Poisson pencil (1) it is possible to introduce it by means of the mentioned above substitution $a_{i}^{j} \rightarrow a_{i}^{j}+\lambda \delta_{i}^{j}$. Under this substitution the space $I_{-}^{q}$ transforms to the space

$$
\begin{gathered}
J^{h, q}=\operatorname{Span}\left(a_{k}^{i} a_{k}^{j}-q a_{k}^{j} a_{k}^{i}-h\left(\delta_{k}^{i} a_{k}^{j}+a_{k}^{i} \delta_{k}^{j}\right),\right. \\
a_{i}^{k} a_{j}^{k}-q a_{j}^{k} a_{i}^{k}-h\left(\delta_{i}^{k} a_{j}^{k}+a_{i}^{k} \delta_{j}^{k}\right), i<j ; a_{i}^{l} a_{k}^{j}-a_{k}^{j} a_{i}^{l}=0, \\
\left.a_{i}^{j} a_{k}^{l}-a_{k}^{l} a_{i}^{j}-\left(q-q^{-1}\right) a_{k}^{j} a_{i}^{l}-h m\left(\delta_{i}^{l} a_{k}^{j}+a_{i}^{l} \delta_{k}^{j}\right), i<k, j<l\right),
\end{gathered}
$$

where $h=\lambda(q-1), m=1+q^{-1}$.

Then setting $A_{h, q}=T(W) /\left\{J^{h, q}\right\}$ we obtaine by construction a flat deformation of the algebra $A_{0, q}$.

Thus we have quantized the both types of Poisson pencils under consideration and introduced the corresponding quantum algebras. In next Section we discuss the Lie algebra-like objects generating these quantum algebras.

\section{Generalized Lie algebras and flatness of de- formation}

It is well-known that the axioms system of the ordinary Lie algebras $\mathfrak{g}$ allows one

1. to prove the PBW theorem and therefore to establish a flatness of deformation from the symmetric algebra $\operatorname{Sym}(\mathfrak{g})$ to the enveloping one $U(\mathfrak{g})$;

2 . to introduce a notion of $\mathfrak{g}$-module and to construct a tensor category of $\mathfrak{g}$-modules using the Hopf structure of the algebra $U(\mathfrak{g})$. 
In this Section we discuss a possible generalization of the notion of a Lie algebra in the connection with flatness of deformations discussed above.

Let $V$ be a linear space and let $I$ be an arbitrary subspace in $V^{\otimes 2}$. Given a linear morphism $[]:, I \rightarrow V$, it is natural to introduce its "enveloping algebra" as follows

$$
A=T(V) /\{\operatorname{Im}(i d-[,]) I\} .
$$

(Let us note that in the classical case, i.e., when [,] is an ordinary Lie bracket, our definition of the enveloping algebra differs from the usual one by the factor 2 in the bracket.)

This algebra is a filtered quadratic one. We consider its adjoint graded algebra $\operatorname{Gr} A$. It is evident that if there exists a graded isomorphism of the PBW type $\operatorname{Gr} A \rightarrow T(I) /\{I\}$ then the following relations are satisfied

$$
\begin{gathered}
\left([,]^{12}-[,]^{23}\right)(I \otimes V \cap V \otimes I) \subset I, \\
{[,]\left([,]^{12}-[,]^{23}\right)(I \otimes V \cap V \otimes I)=0 .}
\end{gathered}
$$

If the g.q.a. $T(I) /\{I\}$ is Koszul (cf. $\mathbb{P P}]$ ) then a reciprocal statement is true as well.

Proposition 3 ([PP], BG] Given a linear space $V$, a subspace $I \subset V^{\otimes 2}$ and an operator ("Lie bracket") [, ] :I $\rightarrow V$. Then if the g.q.a. $T(V) /\{I\}$ is Koszul and the relations (7), (8) are fulfilled then there exists a graded isomorphism $\operatorname{Gr} A \rightarrow T(V) /\{I\}$.

Let us point out that in this Proposition the bracket [, ] is defined only on the subspace $I \subset V^{\otimes 2}$. However in the cases under consideration we always have a pair of subspaces $I_{ \pm}$or $\bar{I}_{ \pm}$(the space $I_{-}$or $\bar{I}_{-}$plays the role of the space $I$ from Proposition 4). This motivated us to introduce the following

Definition 1 The data $\left(V, V^{\otimes 2}=I_{+} \oplus I_{-},[]:, V^{\otimes 2} \rightarrow V\right)$ is called a generalized Lie algebra if the following system of axioms is fulfilled

1. $[,] I_{+}=0$;

2. the relations (7), (8) take place with $I=I_{-}$;

3. the g.q.a. $T(V) /\left\{I_{-}\right\}$is Koszul.

Let $\mathfrak{A}$ be a category. Then we say that a generalized Lie algebra is a $\mathfrak{A}$-Lie algebra if one more axiom is fulfilled

4. the spaces $V, I_{ \pm}$are objects of the category $\mathfrak{A}$ and the bracket [, ] is a morphism of this category. 
For the first time a definition of such a type was given in [G4].

The space $V$ equipped with a structure of a generalized Lie algebra will be denoted by $\mathfrak{g}$ and the enveloping algebra (6) will be denoted by $U(\mathfrak{g})$.

Note that in the above definition we don't use any quantum R-matrix. We formulate this definition in terms of two subspaces in the space $V^{\otimes 2}$ but actually we use a quantum R-matrix to define the mentioned subspaces in one or another way.

Note also that, since in frames of our construction (compared with one from Proposition 3) the bracket [, ] is defined on the whole space $V^{\otimes 2}$, we can introduce an analog of the adjoint operators by setting

$$
x \in V \rightarrow a d_{x}: V \rightarrow V \text { where } a d_{x} y=[x, y] .
$$

A way to extend such operator to a "coadjoint vector field" is discussed in [DG2].

Proposition 4 The algebras $A_{0, q}$ for generic $q$ are Koszul for the both types of the above Poisson pencils.

It is so since the algebras $A_{0,1}$ are Koszul (for the fisrt type algebras this was proved in $[\mathrm{B}]$ and for the second type ones it is well known) and the fact that the deformation from $A_{0,1}$ to $A_{0, q}$ is flat (cf. []PP] for detail).

Thus the axiom 3 from the above definition is satisfied.

Using this fact we can state that the deformation from $A_{0,1}$ to $A_{h, q}$ is flat iff the q-bracket satisfies the relation (7) and (8). Assuming this, (for the case $\mathfrak{g}=s l(2)$ this fact was proved in [DG1]), we have by the construction a $\mathfrak{A}$-Lie algebra where $\mathfrak{A}$ is the category of $U_{q}(\mathfrak{g})$-modules.

As for generalized Lie algebras connected to the second type quantum algebras $A_{h, q}$ it is natural to introduce the corresponding bracket by setting $[,] I_{+}=0$ and

$$
\begin{gathered}
{[,]\left(a_{k}^{i} a_{k}^{j}-q a_{k}^{j} a_{k}^{i}\right)=h\left(\delta_{k}^{i} a_{k}^{j}+\delta_{k}^{j} a_{k}^{i}\right),[,]\left(a_{i}^{k} a_{j}^{k}-q a_{j}^{k} a_{i}^{k}\right)=h\left(\delta_{j}^{k} a_{i}^{k}+\delta_{i}^{k} a_{j}^{k}\right), i<j} \\
{[,]\left(a_{i}^{l} a_{k}^{j}-a_{k}^{j} a_{i}^{l}\right)=0,[,]\left(a_{i}^{j} a_{k}^{l}-a_{k}^{l} a_{i}^{j}-\left(q-q^{-1}\right) a_{i}^{l} a_{k}^{j}\right)=} \\
m h\left(\delta_{k}^{j} a_{i}^{l}+\delta_{i}^{l} a_{k}^{j}\right), i<k, j<l .
\end{gathered}
$$

It is evident by the construction that the relation (7) and (8) are satisfied. Thus the latter bracket defines in the space $W$ a structure of a generalized Lie algebra indeed. 
Note that the enveloping algebra of a generalized Lie algebra does not have in general any "generalized bialgebra structure" and therefore it is not possible to define a tensor product of two modules over this associative algebra in the category of $U(\mathfrak{g})$-modules. Let us precise ungoing into details that under "generalized bialgebra structure" we mean a bialgebra structure which is defined in a tensor or quasitensor category. Certain algebras of such a type (called braided groups) were constructed by Sh. Majid but his braided groups arising from the quantum group $U_{q}(\mathfrak{g})$ are not deformational objects (in particular, it is not possible to define the corresponding Poisson bracket).

However there exists a class of generalized Lie algebras whose enveloping algebras admit "generalized bialgebra structures", namely S-Lie algebras (introduced in [G1] under the name of generalized Lie algebras). Their enveloping algebras are objects of the symmetric tensor categories (for these categories the "commutativity morphism" $S$ is involutive: $\left.S^{2}=i d\right)$. We refer the reader to GRZ for a definition of $S$-Lie algebras.

Completing this Section we want to compare our definition of a generalized Lie algebra with close notions introduced by other authors.

Let us first note that construction of the "quantum Lie algebras" from [M] is based on the following fact. There exists a subspace $L$ in the quantum group $U_{q}(\mathfrak{g})$ closed with respect to the quantum adjoint action of $U_{q}(\mathfrak{g})$ on itself. Then a quantum Lie structure on $L$ is defined by the restriction of this action on $L$. A similar construction is given in DOS (cf. also the references therein) where a definition of the enveloping algebra of such type algebras is discussed as well. However the latter algebra is not a flat deformation of any classical counterpart.

The same feature is inherent in the so-called "braided Lie algebras" from [M] (whose enveloping algebras are discussed in [M] as well). The definition of such a Lie algebra-like object consists in a linear space beloning to a braided category and equipped with three operators: a Lie bracket, a coassociative coproduct and a counit which satisfy some axioms.

In contrary, we use only an operator of a Lie bracket in our definition but we impose the axiom of Koszulity to ensure flatness of the deformation of the enveloping algebra.

Let us emphasize that the most controversial question in all attempts to introduce Lie algebras-like structures is a "reasonable" form of the Jacobi identity.

It is worth to note that in the general case we can not reduce the difference 
between two brackets $[,]^{12}-[,]^{23}$ in the relations $(7)-(8)$ to one of them. It is possible only for $S$-Lie algebras whose Jacobi identity has more familiar form

$$
[,][,]^{12}\left(i d+S^{12} S^{23}+S^{23} S^{12}\right)=0
$$

or

$$
[,][,]^{12}=[,][,]^{23}\left(i d-S^{12}\right) .
$$

Under the latter form the Jacobi identity has appeared in the paper [W] in the frames of the quantum differential calculus. However the quantum differential calculus of Woronowicz is not a result of any flat deformation of the ordinary one and therefore the latter form of Jacobi identity is not motivated by the deformation theory (although the basic object of the quantum differential calculus, the algebras of quantized function $F u n_{q}(G)$, has a deformational nature and arises as a quantization of a Poisson bracket).

\section{Example: $n=2$ (type 2)}

Consider the algebra $\operatorname{Mat}(2)$ consisting of the matrices $\left(\begin{array}{ll}a & b \\ c & d\end{array}\right)$. The space Fun(Mat(2)) is generated by the matrix elements $a, b, c, d$. The bracket $\{,\}_{2}$ in this case is determined by the following multiplication table

$$
\begin{gathered}
\{a, b\}_{2}=a b,\{a, c\}_{2}=a c,\{a, d\}_{2}=2 b c \\
\{b, c\}_{2}=0,\{b, d\}_{2}=b d,\{c, d\}_{2}=c d .
\end{gathered}
$$

The linearization of this bracket gives rise to the linear bracket $\{,\}_{1}$ :

$$
\begin{aligned}
& \{a, b\}_{1}=b,\{a, c\}_{1}=c,\{a, d\}_{1}=0, \\
& \{b, c\}_{1}=0,\{b, d\}_{1}=b,\{c, d\}_{1}=c .
\end{aligned}
$$

Let us describe now the corresponding quantum algebras. First we consider the space $W=\operatorname{Span}(a, b, c, d)$ equipped with the operator

$$
S_{W}=S \otimes S^{-1}: W^{\otimes 2} \rightarrow W^{\otimes 2} .
$$


where the solution $S$ of the QYBE has the following form

$$
\left(\begin{array}{cccc}
q & 0 & 0 & 0 \\
0 & q-q^{-1} & 1 & 0 \\
0 & 1 & 0 & 0 \\
0 & 0 & 0 & q
\end{array}\right)
$$

Let us represent now the space $W^{\otimes 2}$ as a direct sum of two subspaces

$$
\begin{gathered}
I_{-}^{q}=\operatorname{Span}\left(a b-q b a, a c-q c a, b d-q d b, c d-q d c, b c-c b, a d-d a-\left(q-q^{-1}\right) c b\right), \\
I_{+}^{q}=\operatorname{Span}\left(a^{2}, b^{2}, c^{2}, d^{2}, q a b+b a, q a c+c a, q b d+d b,\right. \\
\left.q c d+d c, a d+d a, b c+c b+\left(q-q^{-1}\right) a d\right),
\end{gathered}
$$

The algebra $A_{0, q}=T(W) /\left\{I_{-}^{q}\right\}$ is a deformation of the symmetric algebra of the space $W$ "in direction" of the bracket $\{,\}_{2}$. Now we deform the latter algebra in the second time introducing the algebra $A_{h, q}$ as the quotient algebra of $T(V)$ over the ideal generated by the elements

$$
\begin{gathered}
a b-q b a-h b, a c-q c a-h c, b d-q d b-h b, \\
c d-q d c-h c, b c-c b, a d-d a-\left(q-q^{-1}\right) c b .
\end{gathered}
$$

Let us describe the space $I_{-}^{q} \otimes W \cap W \otimes I_{-}^{q}$ explicitly. It is generated by the following four elements

$$
\begin{gathered}
(a b-q b a) c-(a c-q c a) b+q^{2}(b c-c b) a=a(b c-c b)-b(a c-q c a)+q c(a b-q b a), \\
(a b-q b a) d-q\left(a d-d a-\left(q-q^{-1}\right) c b\right) b+q(b d-q d b) a+\left(q^{2}-1\right)(b c-c b) b= \\
a(b d-q d b)-q b\left(a d-d a-\left(q-q^{-1}\right) c b\right)+q d(a b-q b a), \\
(a c-q c a) d-q\left(a d-d a-\left(q-q^{-1}\right) c b\right) c+q(c d-q d c) a= \\
a(c d-q d c)-q c\left(a d-d a-\left(q-q^{-1}\right) c b\right)+q d(a c-q c a)+\left(q^{2}-1\right) c(b c-c b), \\
(b c-c b) d-q(b d-q d b) c+q(c d-q d c) b=b(c d-q d c)-c(b d-q d b)+q^{2} d(b c-c b) .
\end{gathered}
$$

It is instructive to check by direct computations that the relations (7) and (8) are satisfied. Let us introduce now a generalized Lie bracket accordingly to the above scheme by setting

$$
[,] I_{+}=0,[,](q a b-b a)=h b, \ldots,[,]\left(a d-d a+\left(q-q^{-1}\right) c b\right)=0
$$


From this one can immediately deduce the following multiplication table for the bracket $[$,$] :$

$$
\begin{gathered}
{[a, a]=[b, b]=[c, c]=[d, d]=[a, d]=[d, a]=[b, c]=[c, b]=0,[a, b]=[b, d]=M b} \\
{[b, a]=[b, d]=-M q b,[a, c]=[c, d]=M c,[c a]=[d, c]=-M q c}
\end{gathered}
$$

with $M=h\left(1+q^{2}\right)$.

The reader can readily compare this table with one for the "braided Lie algebra $\operatorname{sl}(2)$ " from [DG1].

Completing the paper we would like to stress once more that the second type Poisson bracket $\{,\}_{2}$ is not compatible with one $\{,\}_{g l}$. The reader can easily to check this for the case $n=2$ observing that

$$
\left\{a,\{b, d\}_{g l}\right\}_{2}+\left\{a,\{b, d\}_{2}\right\}_{g l}+c . p . \neq 0
$$

(general case follows from this one). Therefore any reasonable (in the above sense) deformation of Lie algebra $g l(n)$ does not exist in the frame of the second type construction.

Acknowledgements. The authors want to thank Mathematical Departement of Université Claude Bernard, Lyon 1, where they begin to work on the paper. They are also acknowledged the hospitality of Centre de Mathématique de l'Ecole Polytechnique where the final version of the paper was prepared. V.R. was partially supported by RFFR-MF

\section{References}

[B] R.Bezrukavnikov Koszul property of algebra of functions on the minimal orbit, Preprint, Brandeis University

[BG] A.Braverman, D.Gaitsgory Poincaré-Birkhoff-Witt theorem for quadratic algbras of Koszul type, hep-th/9411113

[DG1] J.Donin, D.Gurevich Braiding of the Lie algebra sl(2), in Advances in Soviet Mathematics, V.Lychagin, Editor, Providence R.I., to appear

[DG2] J.Donin, D.Gurevich Quantum orbits of R-matrix type, hepth/9411034 
[DGM] J.Donin, D.Gurevich, Sh.Majid R-matrix brackets and their quantisation, Ann. de l'Institut d'Henri Poincaré, 58 (1993), pp.235-246

[DS1] J.Donin, S.Shnider Quasi-associativity and flatness criteria for quadratic algebra deformation, Israel Math. J., to appear

[DS2] J.Donin, S.Shnider Quantum symmetric spaces, hep-th/9412031,

[DOS] B.Drabant, O.Ogievetsky, M.Schlieker Cohomology of quantum enveloping algebras, Preprint MPI-Ph/93-57, LMU-TPW 1993-19.

[D] V.Drinfeld Hamiltonian structures on Lie groups, Lie bialgebras and the geometric meaning of the classical Yang-Baxter equations, Soviet Math,Dokl.27(1983),pp.68-71

[G1] D.Gurevich Generalized translation operators on Lie groups, Soviet J. Contemporary Math. Anal. 18 (1983), pp.57-70

[G2] D.Gurevich Hecke symmetries and quantum determinants, Soviet Math. Dokl., 38 (1989), no.3, pp.555-559

[G3] D.Gurevich Algebraic aspects of the quantum Yang-Baxter equation, Leningrad Math.J. 2 (1991), pp.801-828

[G4] D.Gurevich Hecke symmetries and braided Lie algebras, in: Spinors, Twistors, Clifford Algebras and Quantum Deformation, 1993, Kluwer Academic Publishers, pp.317-326

[GRZ] D.Gurevich, V.Rubtsov, N.Zobin Quantization of Poisson pairs: $R$ matrix approach, JGP 9 (1992), pp.25-44

[GP] D.Gurevich, D.Panyushev On Poisson pairs associated to modified R-matrices, Duke Math. J. 73 (1994), pp.249-255

[LT] G.Lancaster, J.Towber Representation-Functor and Flag-algebras for the Classical Groups 1, Journal of Algebra 59 (1979), pp.16-38

[M] Sh.Majid Quantum and braided Lie algebras, Journ.Geom.Phys. 13(1994),pp.307-356. 
[RTF] N.Reshetikhin, L.Takhtadzhyan, L.Faddev Quantization of Lie groups and Lie algebras, Leningrad Math.J. 1 (1990), pp.193-226

[PP] A.Polishchuk, L.Positcelski Quadratic algebras, Preprint, 1994

[S] M.Semenov-Tian-Shansky What is classical r-matrix?, Funct. Anal. Appl. 17 (1983), pp.259-272.

[W] S.L.Woronowicz Differential calculus on compact matrix pseudogroups (quantum groups), CMP 122 (1989), pp.125-170. 